\title{
La relación lenguaje y conocimiento y su aplicación al aprendizaje escolar
}

\section{The relationship between language and knowledge and its application to school learning}

\author{
Ángela Camargo Uribe ${ }^{1}$ \\ Christian Hederich Martínez²
}

\section{Resumen}

Este artículo expone tres aproximaciones teóricas que han sido propuestas para la comprensión de la relación entre el lenguaje y conocimiento: la del cognitivismo, la del socio constructivismo y la del socio constructivismo discursivo. Posteriormente, presenta secuencias de aula que ilustran cada uno de estos enfoques, a fin de analizar y evaluar cada postura desde el punto de vista del aprendizaje escolar. Finalmente, llega a conclusiones sobre las relaciones entre los tres enfoques considerados respecto de su aplicación específica al contexto pedagógico y didáctico.

Palabras clave:

Lenguaje, conocimiento, aprendizaje escolar, discurso, comunicación, aula de clase.

Abstract

This paper explains three theoretical approaches that have been proposed to explain the relationship between language and knowledge: cognitivism, social constructivism and discursive constructivism. Later on, the paper presents different classroom dialogues and speech sequences that illustrate each approach to analyze and evaluate them from the point of view of school learning. Finally, some statements are about the relationships among the three approaches and with respect to their application to the classroom context.

Keywords:

Language, cognition, school learning, discourse, communication, classroom

Artículo recibido el 27 de febrero de 2010 y aprobado el 30 de abril de 2010

1 Profesora Universidad Pedagógica Nacional. Estudiante del Doctorado Interinstitucional en Educación de la misma Universidad. Miembro del Grupo de Investigación en Estilos Cognitivos. acamargo@pedagogica.edu.co

2 Profesor Universidad Pedagógica Nacional. Doctor en Psicología. Coordinador del Grupo de Investigación en Estilos Cognitivos. hederich@pedagogica.edu.co 


\section{Introducción}

Tanto en el ámbito de las propuestas psicológicas sobre el conocimiento, como en el de la reflexión lingüística respecto de la función cognitiva del lenguaje, se reconoce la relación entre lenguaje y cognición como estrecha y necesaria (Halliday, 1993., Bruner, 1997., Mercer, 2001., Chomsky, 2002., Bronckart, 2005). La universalidad de esta idea no ha impedido, sin embargo, la aparición de planteamientos diversos sobre la naturaleza exacta de tal relación. Una revisión general a propuestas teóricas e investigaciones sobre el tema, permite identificar al menos tres posiciones claramente diferentes respecto de la forma como el lenguaje se relaciona con la construcción del conocimiento3:

1. El lenguaje como representación, y en este caso, responde a los planteamientos del cognitivismo respecto de la necesidad de códigos simbólicos para expresar el conocimiento (p.ej., Pavio, 1986).

2. El lenguaje como comunicación, y de esta manera, es coherente con la perspectiva del constructivismo social en la que el lenguaje es el puente entre el conocimiento compartido y el conocimiento personal (p.ej., Edwards y Mercer, 1988).

3. El lenguaje como actividad social y cognitiva de producción de sentido, en la que tal y como lo pregona el constructivismo discursivo, la acción lingüística es, de hecho, una actividad de aprendizaje (p.ej., Martin, 1993., Bronckart, 2005).

Estas funciones del lenguaje, en el proceso de construcción de conocimiento, dejan ver la complejidad de la relación de la que estamos hablando. Esta complejidad es consecuencia, a nuestro juicio,

3 Es importante aclarar aquí que el concepto de lenguaje se manejará en este escrito en su acepción más amplia, es decir, como la facultad humana para la signific ación y la comunic ación. Así, el término "lenguaje", si bien la mayor parte de las veces corresponderá al código lingüístico verbal, también llamado "lengua", en algunas ocasiones el término cubrirá eventos semióticos no verbales tales como los sistemas de representación iconográfica, los códigos de notación matemática o química, etc. de una condición del lenguaje humano que lo hace conjugar, sin contradicción alguna, ¿simultáneamente, las tres funciones complementarias antes mencionadas.

Cada una por aparte, las tres teorías tienen amplios desarrollos conceptuales, argumentativos e investigativos que apoyan sus planteamientos; y que bien estudiadas, cada una de ellas, son claramente plausibles. Revisadas en el orden en que se presentan podrían incluso decirse que van abarcándose consecutivamente de manera tal que la segunda cubre la primera y la última comprende las dos anteriores.

Ahora bien, si se considera el contexto del aprendizaje escolar, un contexto en el que el uso del lenguaje oral y escrito es prácticamente omnipresente, el asunto de la relación entre lenguaje y conocimiento adquiere no sólo relevancia teórica, sino importancia pedagógica e incluso didáctica. Cabe entonces la pregunta: ¿pueden trasladarse estos planteamientos teóricos a la situación específica del aprendizaje escolar? Intentaremos responder a esta pregunta en este trabajo.

El presente escrito tiene entonces el propósito de a) ilustrar la manera como cada uno de los enfoques explicativos de la relación entre lenguaje y conocimiento adquiere forma en el aula de clase e b) identificar las posibilidades de cada enfoque para el propósito final del aprendizaje escolar.

\section{Hacia una metodología que haga visible el papel del lenguaje en el aprendizaje escolar}

Es innegable la constante presencia del lenguaje en los diversos momentos del aprendizaje escolar. Podría agregarse además, que la comprensión de su papel durante el aprendizaje es de vital importancia en la búsqueda de soluciones a problemas tales como el fracaso escolar o los bajos niveles de logro académico.

Adquiere sentido en este punto la búsqueda de estrategias metodológicas que hagan visible y comprensible la relación entre lenguaje y conocimiento para la situación del aula de clase. Una de las estrategias que mayor acogida ha tenido en el contexto de las investigaciones sobre la enseñanza y el aprendizaje en el contexto escolar es la ob- 
servación micro-etnográfica de las interacciones comunicativas que ocurren en el aula (p.ej., Green y Harker, 1982., Stubbs, 1983).

En los años 80 y principios de los 90, luego de un largo periodo en el cual el estudio científico de los fenómenos lingüísticos y comunicativos tuvo como énfasis la dimensión formal -significante- de la lengua (Matthews, 1983., Halliday, 1984), se comenzó a virar hacia el reconocimiento, exploración y descripción de dimensiones de los sistemas de las lenguas imposibles de ser descritas (al menos plenamente) en independencia de su contexto de uso: los planos semántico y comunicativo del lenguaje (Levinson, 1985., Leech, 1983., Escandell, 1993). Ello llevó a la aparición de metodologías y técnicas etnográficas -etnografías del habla o de la comunicación- para la recolección y el análisis lingüístico. Estas metodologías consideraban el fenómeno del lenguaje desde el punto de vista de su uso en situaciones de acción e interacción comunicativas. Una de las situaciones de uso lingüístico que ha sido objeto de atención por parte de los etnógrafos del habla es la situación del salón de clase (Wilkinson, 1982., Susan Ervin Tripp, 1982).

De acuerdo con Nussbaum y Tusón (1996), estos desarrollos de la investigación lingüística contribuyeron al inicio de una línea de investigación en pedagogía, psicología y sociología educativas, que considera el aula "como el locus apropiado dónde centrar la atención para entender los complejos mecanismos que subyacen a la enseñanza y al aprendizaje". La mirada a la situación de la clase escolar desde el punto de vista comunicativo ha tenido avances recientes en el ámbito de las investigaciones en didácticas específicas. Tal es la propuesta de la metodología en clínica didáctica como modelo de investigación desarrollado por Rickenmann y sus colegas de la Universidad de Ginebra, y aplicado a eventos de enseñanza y aprendizaje de las artes, las ciencias naturales y las matemáticas (Rickenmann, 2007).

Siguiendo de manera general los lineamientos metodológicos de la etnografía del habla, lo que sigue a continuación es una exploración de las tres perspectivas expuestas sobre la naturaleza de la relación entre lenguaje y aprendizaje, a fin de identificar su presencia en las interacciones discursivas y comunicativas del aula de clase.

Con tal fin, se contará con observaciones hechas en el contexto de la investigación doctoral que actualmente estamos desarrollando. El proyecto trata, a grandes rasgos, de los procesos discursivos y comunicativos presentes en el aula de clase, vistos ellos desde el punto de vista del profesor. Aunque con metas diferentes, pues nuestro propósito en el trabajo doctoral es la identificación de estilos de enseñanza desde la dimensión comunicativa de la labor docente, el corpus recolectado hasta el momento se adecúa a los objetivos de este ejercicio reflexivo, pues corresponde a filmaciones de clases de ciencias naturales dirigidas a estudiantes de educación básica secundaria (6to a 8vo grados) y conducidas por profesores de instituciones educativas del sector oficial de la ciudad de Bogotá4.

Los eventos comunicativos registrados y observados serán el substrato para la exploración planteada. Así, los ejemplos que se utilizarán aquí se relacionan con el aprendizaje de las ciencias naturales. Desde nuestro punto de vista, ello no obsta para que las conclusiones a las que se llegue no sean aplicables al aprendizaje de cualquier otro tipo de contenidos, al menos en lo que toca a contenidos de aprendizaje de tipo declarativo.

\section{Primera aproximación: la representación lingüística del conocimiento ya construido}

Esta primera aproximación corresponde a la visión cognitivista en psicología del conocimiento en la que éste, se expresa mediante el lenguaje (Eysenck y Keane, 1990; Best; 1999). Desde este punto de vista, el conocimiento surgiría primero en el individuo en una especie de estado puro para luego poder ser comunicado o expresado mediante procesos de simbolización (codificación de un estado psicológico preexistente). El lenguaje humano sería un vehículo

4 Se trata de la investigación doctoral titulada Dimensiones cognitiva, comunicativa y lingüística del estilo de enseñanza, realizada por Ángela Camargo, bajo la dirección del profesor Christian Hederich Ph.D, en el marco del Doctorado en Educación de la Universidad Pedagógica Nacional. 
de codificación, registro y almacenamiento de algo que ya existe en independencia del medio en el que se expresa. Subyacente a tal planteamiento hay un énfasis en el lenguaje desde el punto de vista de su función formal representacional, en la que se reconoce a la cognición humana como eminentemente simbólica (Fodor, 1997, 2000., Chomsky, 2002).

Estos planteamientos, indicadores de la tradición cartesiana que enmarca al formalismo cognitivista, suponen que, una vez se ha logrado su aprendizaje, el estudiante muestra o demuestra lo logrado mediante su expresión en códigos lingüísticos verbales o no verbales; en todo caso, adecuados al contenido de lo aprendido (Rumelhart y Ortony, 1977., Pavio, 1986). En esta medida, resulta comprensible que las evaluaciones escolares del aprendizaje se realicen utilizando la mediación lingüística, usualmente el rígido registro académico de la lengua escrita. También en consonancia con ello, múltiples actividades escolares tienen como función que el estudiante perciba la necesidad de "empaquetar" el conocimiento aprendido en algún código (verbal o no verbal) convencionalmente reconocido como el adecuado para el contenido específico del aprendizaje (Schank y Abelson, 1977., Gutiérrez-Rodilla, 1998).

En efecto, el enfoque representacional es consecuente con el hecho de que cada asignatura que se aprende posee sus propios sistemas de representación que acompañan la variedad o registro lingüístico especializado: tablas, gráficas, mapas, alfabetos, formulas, nomenclaturas, secuencias, etc. Es frecuente observar, por ejemplo, que el profesor en su discurso, llame la atención de los estudiantes sobre cómo y para qué utilizar ciertas formas de representación del conocimiento. Un ejemplo extraído del corpus ilustra este gesto profesional del docente:

En la situación que vamos a considerar (Atenea, $3,7)^{5}$, la profesora está haciendo una explicación

5 Este rótulo se refiere a la forma como cada clase ha sido codificada. El nombre corresponde al código del profesor específico observado, el primer número corresponde al número de la clase del conjunto que se filmó con un profesor en particular, y el segundo corresponde al grado escolar en el que están los niños (sexto, séptimo u octavo). sobre la organización molecular de la materia. Comenzado con el concepto de organismo, ella pone en marcha un juego de pregunta-repuesta del tipo: “¿De qué está hecho...?”. De esta forma, ella y los estudiantes pasan del organismo al sistema, del sistema al órgano, del órgano al tejido y del tejido a las células. A medida que aparece un nuevo nivel de composición de la materia, ella dibuja en el tablero cada elemento (un hombre, un aparato digestivo, un estómago, un tejido estomacal, una célula). En el momento en que llega a la pregunta:

P6. ¿Y las células...?

E. Cromosomas, cromosomas

E. Moléculas, profe

P ¡Moléculas! (Escribe la palabra en el tablero). Las moléculas que forman las células son moléculas gigantes. Entonces yo no voy a hacer ningún dibujo, yo no sé dibujar una molécula de $\mathrm{ADN}$, ni una proteína gigante, ni una célula adiposa gigante... ¿Y las moléculas?

E: De átomos

P: De átomos... Y como estoy haciendo dibujos, entonces tocaría pensar en cómo hacer un dibujo de un átomo... Como los átomos no los podemos ver...

E. El núcleo

E. Una bolita

P. Los átomos no los podemos ver, pero podemos ver los dibujos que hacen en los libros... Como por ejemplo una bolita roja... una bolita azul, se llaman ¡modelos! Se llaman modelos, modelos de átomos. Se pueden hacer... representar las moléculas o los átomos utilizando modelos... Y como vamos hablar de las moléculas, entonces digamos... llamémoslas modelos moleculares. Los modelos moleculares son representaciones imaginarias del interior de la materia. Representaciones imaginarias porque no las estamos viendo, pero para entender bien. Pero para entender bien

6 Los enunciados encabezados con la letra P son emitidos por el profesor y los enunciados encabezados con la letra $E$ son emitidos por estudiantes. 
cómo es la estructura interna de la materia hacemos entonces representaciones imaginarias de las moléculas y de los átomos. Por ejemplo, una forma que ustedes han visto en los libros son las esferas de colores o en los libros se ven dibujados círculos de colores.

Toda esta secuencia de clase tiene que ver con el manejo de códigos para expresar el conocimiento que la ciencia ha construido. En un primer momento, la profesora centra la atención en dos sistemas semióticos usados para expresar un saber muy particular: el iconográfico y el léxico. Por un lado, está el sistema iconográfico o los dibujos esquemáticos que se usan para ilustrar y al tiempo representar componentes de la materia. Se trata de dibujos poco elaborados (no es una clase de arte), pero lo suficientemente completos como para que se identifique lo que se desea representar. No contenta con ello, la profesora rotula los dibujos con sus nombres, es decir, utiliza el recurso lingüístico léxico como un segundo sistema de representación. Esta práctica de uso redundante en el que se usan varios códigos (palabra coloquial, palabra técnica y formula química) para un mismo referente es común en la expresión científica y obedece a una búsqueda de precisión que privilegia la claridad sobre criterios de forma, por ejemplo la repetición.

Esta secuencia de pasar del dibujo a la palabra llega hasta cuando se introduce el nivel de las moléculas. En este punto, la profesora comienza a abandonar el código iconográfico: "yo no voy a hacer ningún dibujo, yo no sé dibujar una molécula de ADN, ni una proteína gigante, ni una célula adiposa gigante", y utiliza solo el léxico (escribe en el tablero la palabra molécula). Finalmente, llega al nivel de los átomos y dice "tocaría pensar en cómo hacer un dibujo de un átomo... Como los átomos no los podemos ver...".

Descartado el código iconográfico como recurso posible para representar los átomos que componen las moléculas, se crea el contexto propicio para proponer el código de los modelos moleculares. Además de presentar el código (esferas o círculos de diferentes tamaños y colores), la profesora intro- duce el concepto de modelo molecular y explica la necesidad de proponer esta forma de representación, más abstracta que la iconográfica. Así, la profesora está introduciendo la necesidad de utilizar códigos para darle forma al conocimiento abstracto: "para entender bien cómo es la estructura interna de la materia hacemos entonces representaciones imaginarias de las moléculas y de los átomos".

El ejemplo anterior ilustra un aspecto del aprendizaje no siempre entendido en los contextos escolares. Además de lo que podría llamarse el contenido del aprendizaje es necesario aprender su forma, es decir, su representación semiótica en códigos construidos específicamente para ello. En realidad, podría afirmarse que este doble aprendizaje (contenido-forma) es universal en los procesos pedagógicos y didácticos ocurridos en el aula. Incluso está presente en situaciones en donde esta doble dimensión del aprendizaje no es tan evidente, puesto que la parte formal de la díada corresponde a una variedad especializada de la lengua, una jerga. Hablamos aquí del discurso disciplinar: el discurso académico. A pesar de que se trata de una lengua común a todos, el aula de clase trabaja con el discurso disciplinar de la misma manera que si fuera un código totalmente desconocido por el alumno que el profesor está enseñando.

En la siguiente secuencia de clase (Artemisa, 2, 7), la profesora está realizando la actividad de lectura de un texto sobre el tema de la biodiversidad. En los turnos asignados por ella, los niños van leyendo enunciados del texto. El diálogo se sucede así:

E: (leyendo) La biodiversidad es quizás el principal parámetro para medir [...] el efecto directo o indirecto de las actividades humanas.

P: Espera un momento. ¿Quién me puede explicar esa frase? Vuelvo y la leo: La biodiversidad es quizá el principal parámetro para medir el efecto directo o indirecto de las actividades humanas en los ecosistemas. ¿Quién me puede explicar esa frase? ¿Cómo la entienden? Cáceres, ¿cómo la entiendes? ¿Te la vuelvo a leer? ¿Te la repito? [...] No se les olvide que estoy haciendo el ejercicio de leer para poder entender de qué me están hablando, ¿cierto? 
¿Cuál es la carreta ${ }^{7}$ que hay ahí? Y eso que solo son dos párrafos. Señor, a ver, aquí, Pérez.

E: Todas las personas toman las medidas exactas para aprovechar los recursos naturales.

P: Mmmm (niega con la cabeza), sigue tirando a frío, si...(señalando a otro niño).

E1: Que según la biodiversidad del ecosistema en el que nos encontremos podemos mirar las reacciones de las actividades humanas.

E2: ¿Qué?

E1: O sea, se puede mirar según la biodiversidad, ¿qué es lo peor para la biodiversidad?; en ese caso...

P: Sí, sí, sí. El impacto que por ejemplo, ocasiona el que aquí en la ciudad, bueno, hay muchos impactos en los que... en los humedales, por ejemplo. ¿Quién está viviendo en los humedales? Las urbanizaciones que están construyendo ahí. ¿Será que se muere algún bicho si hacen alguna construcción en un humedal?

En este apartado la profesora da a entender, mediante su discurso, que un texto científico es algo que se debe descifrar, algo cuya comprensión inicial intuitiva, se debe confrontar con otro código que, en este caso, es el discurso de la cotidianidad: “... estoy haciendo el ejercicio de leer para poder entender de qué me están hablando, ¿cierto? ¿Cuál es la carreta que hay ahi?"

Con su relectura de la frase extraída del manual y sus preguntas sobre cómo entenderla, la profesora está implicando que la comprensión inicial que pudieran haber logrado los estudiantes no basta para dar cuenta del contenido de la expresión. De hecho, el intercambio que surge se asemeja a un proceso de desciframiento en donde la primera interpretación: "todas las personas toman las medidas exactas para aprovechar los recursos naturales" es rechazada por la profesora con la expresión "tirando a frío", típica de un juego de adivinanzas. La segunda: "se puede mirar según la biodiversidad, qué es lo peor para la

7 El término carreta es un colombianismo para expresar el contenido de un discurso con el cual su usuario está involucrado emocionalmente. biodiversidad; en ese caso", en cambio, es aprobada por la profesora.

Podría decirse que lo que se está inculcando es que, para saber ciencias, es necesario saber asignar unos ciertos sentidos a las palabras, diferentes a los que éstas podrían tener en otro contexto. Si bien en un sentido amplio este planteamiento representacional de la relación lenguaje-aprendizaje es válido, representa una concepción bastante reducida de lo que un enfoque lingüístico puede aportar al proceso de aprendizaje. Los dos ejemplos anotados arriba, al tiempo que ilustran la postura, muestran sus peligros a la hora de aplicarla al ámbito escolar.

El primer ejemplo planteado, si bien muestra claramente el enfoque representacional en el aprendizaje, presenta igualmente uno de los peligros que se corre en la aplicación directa de esta postura representacional al salón de clase. Se trata del peligro de caer en el nominalismo como objetivo didáctico. En efecto, la secuencia presentada insinúa una simplificación del asunto de enseñar ciencias a la presentación de un conjunto de términos y sus definiciones, independientemente de las cosas que el estudiante pueda hacer, una vez manejados los rótulos.

El segundo ejemplo, por su parte, muestra otra forma de simplificación del proceso de enseñar ciencias. El ejemplo en cuestión, al tiempo que ilustra la distancia entre un discurso cotidiano y uno académico, indica una estrategia didáctica cuya eficacia es dudosa frente al objetivo de enseñar ciencias. Como se observa, la profesora está implicando (y ese sería el mensaje que deja a sus estudiantes) que el texto científico es algo tan extraño y lejano al mundo de los estudiantes que es necesario gastar mucho tiempo en descifrarlo. Mirada esta estrategia desde el punto de vista de su eficacia para el logro de objetivos de aprendizaje, el aprendizaje de ciencias quedaría reducido a la capacidad de decodificación de un texto, independientemente que aquellas cosas que podrían elaborarse a partir de lo comprendido inicialmente, es decir, independientemente de los niveles de comprensión inferencial que se alcancen.

En consecuencia, a pesar de que la postura representacional del cognitivismo reconoce y da 
cuenta de elementos necesarios para el aprendizaje, en este caso sobre las ciencias parece claro que resulta insuficiente explicarlo frente a los retos que supone la enseñanza escolar. Poder nombrar conceptos en la forma convencional aceptada no implica comprenderlos, sino poderlos usar de forma productiva. Esta idea se encuentra implícita en la aproximación socio- constructivista que ilustramos a continuación.

\section{Segunda aproximación: la construcción de significados compartidos.}

La segunda aproximación corresponde a la perspectiva socio-constructivista de la relación lenguajecognición, representada en las ideas de Vygotsky. Para este autor, las estructuras de conocimiento de un individuo no se desarrollan de manera aislada del contexto de interacción social y cultural en el que crece. En consecuencia, todo aprendizaje tiene lugar primero en el plano social, y allí se constituye en fenómeno inter-psicológico, y luego en el plano psíquico, y es allí donde se constituye en una realidad intra-psicológica (Vygotsky, 1979., Cole, 1985., Rogoff, 1997., Daniels, 2005).

Según este planteamiento, invariablemente, todo conocimiento ha de tener presencia en un contexto social para luego pasar al plano individual $y$, en consecuencia, aprenderse. Este paso es posible gracias al papel de los mediadores socio-culturales, entre los cuales el más importante es el lenguaje. Este último adquiere aquí un estatus algo más protagónico que con los planteamientos cognitivistas iniciales, puesto que la mediación lingüística entre lo externo y lo interno al sujeto debe ocurrir para que ocurra el aprendizaje. El constructivismo social reconoce entonces que la función social comunicativa del lenguaje cumple un papel fundamental en la construcción individual del conocimiento no solo a través de la lengua, sino mediante otras herramientas semióticas que harían igualmente posible el aprendizaje (Gutiérrez-Rodilla, 1998).

En este orden de ideas, construcción del conocimiento escolar, si bien se considera un evento eminentemente individual, se encuentra inserta en actividades de naturaleza colectiva que llevan a cabo profesores y alumnos en un entorno social específico, culturalmente organizado (Candela, 1999., Mercer, 2001., Mortimer y Scott, 2003). En otras palabras, y desde el punto de vista de los actuales modelos inferenciales de la comunicación (p.ej., Sperber y Wilson, 2006), el aprendizaje es posible gracias a que las actividades de interacción comunicativa que ocurren en el aula de clase, conducen a la construcción conjunta de significados.

El planteamiento inferencial reconoce el papel de los lenguajes (códigos o medios semióticos) en la comunicación, pero no considera que ellos sean el elemento fundamental de la misma. Los códigos (verbales o no verbales) sirven como herramientas para construir índices a partir de los cuales se puede inferir el mensaje que el comunicador desea transmitir. Es entonces la inferencia, un proceso cognitivo, el elemento fundamental para que ocurra la comunicación. Como se observa, estamos frente a un modelo de comunicación que reconoce y le asigna especial importancia a la cognición dentro de la comunicación. Cuando dos personas entran en contacto, se ponen en juego dos cerebros, dos mentes, dos sistemas de conocimiento (Sperber, 1994., Sperber y Wilson, 1995). En este sentido, la situación didáctica en la que unos participantes (p.ej., el profesor y sus estudiantes) interactúan con un propósito específico en mente y sobre la base de una serie de supuestos compartidos es un ejemplo típico de situación comunicativa.

En el contexto de la investigación contemporánea en psicopedagogía, autores como Coll (2001) han desarrollado una explicación del aprendizaje escolar que supone, emulando la concepción inferencial de la comunicación, una actividad en la que al menos dos sistemas de conocimientos (conceptos, experiencias, valores) se encuentran, se confrontan, se transforman y se reorganizan a fin de producir aquello que Coll llama "sistemas de significados compartidos".

De acuerdo con este autor, el inicio del proceso de aprendizaje de un contenido curricular específico está caracterizado por encuentros comunicativos en los que profesor y alumnos comparten apenas unos pocos significados (las parcelas de conocimiento se 
encuentran muy separadas entre sí). En consecuencia, las interacciones comunicativas en esta primera etapa del proceso están llenas de inferencias incompletas o equivocadas. Se trata, sin embargo, de una fase necesaria en la que la intención del profesor es construirse una representación del conocimiento de sus alumnos sobre el tema, y la intención de los alumnos es construirse una representación de lo que el profesor quiere que ellos aprendan. Lo que surge aquí de manera conjunta es una serie de objetivos de interacción; unos presupuestos que sirven de base a procesos inferenciales y representacionales posteriores. Durante la consecución de esta plataforma cognitiva compartida desde la cual avanzar en el conocimiento, buena parte de lo que el profesor hace es asegurarse de que él y sus estudiantes manejen el mismo sistema léxico de base. Este gesto del docente asegura que las transacciones posteriores sean comprendidas en el sentido intencionado.

En una segunda etapa, una vez establecidas con claridad las intenciones comunicativas, es decir, los propósitos de aprendizaje, la tarea consiste en hacer evolucionar las representaciones de los alumnos ampliándolas y enriqueciéndolas de forma que el profesor y los alumnos vayan compartiendo progresivamente cada vez más significados relevantes para el contenido de la clase. El reto en este momento es encontrar las interacciones comunicativas adecuadas para evitar, en la medida de lo posible, los malos entendidos, las rupturas y las incomprensiones mutuas, y aclararlas cuando se produzcan.

Si todo va bien, el proceso debe evolucionar lo suficiente como para que, al final del mismo, el sistema de significados que da cuenta de un contenido específico sea el mismo para el profesor y sus alumnos y, en consecuencia, todas las inferencias que se hagan partan de presupuestos conceptuales, actitudinales y pragmáticos comunes.

A fin de ilustrar el proceso que se acaba de explicar, acudiremos a algunas secuencias dialógicas de una clase de octavo grado sobre el tema de la respiración (Tritón, 1,8). En el momento en que el profesor está repasando el tópico de la respiración a nivel celular, se sucede el siguiente intercambio:
E: Difusión, ósmosis, cualquiera de esos dos sistemas. Hay que mirar una cosa. Estos procesos como la ósmosis y la difusión tienen que ver mucho con la concentración. ¿Qué es una concentración de una sustancia? Ahorita me dicen que es una sustancia que piensa mucho, tranquilamente. ¿Sí o no? Concentrados. Cuando una sustancia está concentrada, ¿qué significa?

E: Pesada

E: Espesa.

P: Espesa, bueno, relacionémoslo con espeso. Rodrigo, ¿cómo qué puede ser? [...] ¿Qué es concentración de una sustancia? Me dicen que es espesa. O sea que si yo destapo un tarro de mermelada y está muy espesa, ¿está muy concentrada?

E: Cuando hay mucho de la misma sustancia.

P: Sí, sí... shhh (gesto para pedir silencio), espere, espere que Rodrigo está hablando (se dirige a otro niño que quiere intervenir).

E: Cuando las moléculas están muy pegadas una con otra, entre más pegadas, hay mayor concentración.

P: Está más concentrada, ¿sí? Si relacionamos eso con una mermelada, la mermelada yo la puedo comenzar a hacer en líquido, porque se comienza a hacer en líquido y está bien líquida, pero a medida que va pasando el tiempo y el calor y el calor y el calor, se va concentrando, concentrando, concentrando, hasta que queda la dichosa mermelada en el frasco y espesa. Entonces, sí tiene mucho que ver con concentración.

En su presentación del término "concentración", el profesor primero se asegura de que la palabra en cuestión no sea entendida con acepciones que no vienen al caso, como la de proceso cognitivo (ahorita me dicen que es una sustancia que piensa mucho, tranquilamente. ¿Sí o no?). Una vez establecido el sentido general del término (espesa, bueno, relacionémoslo con espeso), pasa a profundizar en las condiciones físicas de una sustancia para que pueda considerársela concentrada. En términos comunicativos, este intercambio tiene el propósito de sentar bases comunes para la comprensión de explicaciones posteriores en las que es necesario el 
concepto de concentración. Por un lado, y por medio del descarte, el profesor ubica el campo semántico en el que el término debe ser entendido y por otro, se produce una cierta construcción conjunta en la que un estudiante plantea una generalización y el profesor corrobora esta abstracción mediante un ejemplo.

Más adelante en la clase, se produce el siguiente intercambio:

P: ¿Qué pasa con esto? Si yo tengo una célula acá, pongan atención para que no se confundan. Tengo la célula acá (está dibujando en el tablero una célula) [...] Tengo una célula acá, ¿verdad? Y dentro de la célula, ¿hay más oxígeno o menos oxígeno que afuera?

E: Más.

E: Menos.

P: ¿Más? Yo coloco esto así miren (dibuja afuera de la célula el símbolo $\mathrm{O} 2$ en letras grandes) y coloco esto aquí, vean (dibuja adentro de la célula el símbolo $\mathrm{O} 2$ en letras pequeñas). ¿Hay más oxígeno afuera $o$ adentro?

E: Afuera.

P: Afuera, ¿sí o no?

E: Más grande.

P: ¿Cierto? Entonces, el oxígeno pasa de un sitio de mayor concentración a uno de menor concentración. ¿Dónde está más concentrado, afuera o adentro?

E: Adentro.

P: ¿A dónde está más concentrado?

E: Adentro.

P: ¿Afuera o adentro?

E: Adentro.

P: Adentro está más concentrado. ¿Cierto? Entonces, no lo molestemos... (risas y pausa del profesor). $\mathrm{Si}$ les acabo de decir que el sitio donde existe mayor cantidad de cosas es lo que está más concentrado. Si la mermelada se espesó es porque hay una gran cantidad de sustancia ahí, pura, y por eso, espesa, por eso se concentra. ¿A donde hay más concentración? ¿Afuera o adentro?

E: Afuera.
P: Afuera, hay mayor cantidad de oxígeno que aquí adentro. Entonces, las sustancias pasan de un sitio de mayor concentración a uno de menor concentración y van a entrar (escribe en el tablero flechas que van de afuera de la célula a adentro de la célula)...

E: Por los poros van a entrar.

P: Exactamente, van a entrar... esa es la forma en que entra el oxígeno por la membrana celular para llegar al citoplasma de la célula.

La secuencia anterior ilustra con claridad los momentos en los que el intercambio comunicativo no tiene éxito, básicamente, porque las suposiciones del profesor sobre lo que los estudiantes saben, están equivocadas y las suposiciones de los niños respecto de lo que su profesor espera de ellos también lo están.

En efecto, después de haber logrado, no sin esfuerzo, que los alumnos reconocieran que afuera de la célula había más oxígeno que adentro, el profesor no logra que los estudiantes relacionen ese hecho con el paso del oxígeno de zonas de más concentración a zonas de menos concentración. Es claro que el profesor supone que los estudiantes deberían saber que hay menos oxígeno dentro de la célula porque éste se agotó (esta sería la razón por la cual hay menos concentración de oxígeno adentro que afuera) y espera de ellos la respuesta que concuerde con este hecho. Sin embargo, no hay nada en la secuencia de la clase que permita que los estudiantes tomen en cuenta esta información para contestar las preguntas del profesor. Ellos por su parte, parecen haber inferido del diagrama de la célula y de la discusión acerca de la cantidad de oxígeno que el profesor quería puntualizar, especialmente sobre el hecho de que en un espacio pequeño hay más concentración, incluso a pesar de la menor cantidad de oxígeno. Por la reacción del profesor, esta suposición es claramente la equivocada. Vemos pues que la construcción conjunta del proceso de ósmosis no se logra, básicamente por un asunto de comunicación: una pieza de información que uno de los interlocutores pensó que era compartida no lo era y, en su lugar, se construyeron inferencias, co- 
herentes desde el punto de vista lógico, pero inútiles para el logro del aprendizaje.

Es interesante notar que los estudiantes perciben la distancia entre su razonamiento y el del profesor y uno de ellos intenta recuperar la comunión comunicativa al aportar información previamente compartida que él considera relevante: "por los poros van a entrar". Con esta intervención, la comunicación -rota por el malentendido-, se recupera, al menos en parte, a fin de hacer posteriores intentos de pensamiento conjunto.

Por supuesto hay momentos en esta clase en los que el profesor logra una cierta sintonía cognitiva con sus estudiantes. Veamos la siguiente secuencia:

P: Explíquenme los hongos... ellos obtienen todo su alimento de ahí. Al obtener el alimento, ¿qué es lo que están obteniendo?, lo que teníamos acá, ¿qué era? (señala algo que estaba en el tablero, pero que ha sido borrado).

E: Ácido.

E: Glucosa.

P: Glucosa, ¡claro!, obtienen glucosa y a partir de esa glucosa, ¿qué van a obtener?

E: Ácido pirúvico.

P: Y el ácido pirúvico, ¿qué hace?

E: Se convierte en energía.

P: Se convierte en energía, finalmente, ¡exacto!

Como se observa, en esta ocasión el profesor logra una secuencia de aciertos en las respuestas de los estudiantes (glucosa, ácido pirúvico, energía). Desde el punto de vista comunicativo, esto es un indicador de la comunalidad de referentes, en donde las presuposiciones mutuas son compartidas. Desde la mirada socio-constructivista esta fluidez dialógica sería indicadora de que se estaría en el camino hacia el aprendizaje.

Así, desde el punto de vista de la explicación socio-comunicativa del aprendizaje, el reto para el profesor es desarrollar las estrategias y los mecanismos necesarios para que, sobre la base del escaso conocimiento común inicial, todo aquello que él diga vaya adquiriendo el sentido que los objetivos de aprendizaje determinan.

Dentro de los planteamientos del constructivismo social, si el profesor lo hace de una manera sistemática en actividades de interacción conjuntas los estudiantes alcanzarán un desarrollo conceptual que nunca lograrían por sí solos. La función de profesor es la de proporcionar el andamiaje más propicio, en el sentido de Bruner (1973), o operar sobre la base de una zona de desarrollo próximo, a la manera de Vygotsky (Mercer, 2001). La conversación, el diálogo, la discusión, la interacción comunicativa adquiere en este contexto especial relevancia.

Podría decirse que esta visión del papel del lenguaje como actividad comunicativa que conduce hacia el aprendizaje redefine la función de la representación semiótica del conocimiento al incorporar este proceso a un desarrollo mucho más amplio que involucra no sólo la inteligibilidad mutua, sino la producción conjunta de sentido. En esta medida, en contraste con el enfoque cognitivista, el modelo socio- constructivista posee mayor poder descriptivo y explicativo del papel del lenguaje en el aprendizaje.

A pesar de sus logros respecto del enfoque representacional, el planteamiento interactivo de construcción de conocimiento compartido tiene el peligro de mantenerse únicamente en el nivel inter-psíquico y nunca alcanzar la interiorización pretendida. Si repasamos, a manera de ejemplo, los apartes de la secuencia de la clase utilizada -tanto en las ocasiones en que la negociación de sentidos tiene éxito, como en las que no- el peso discursivo recae casi totalmente en el profesor. El papel de los estudiantes, si bien es más activo que la simple repetición de términos, se limita a tratar de inferir lo que el profesor quiere que ellos digan. No se observa entonces una auténtica construcción conceptual del estudiante, puesto que la dinámica de la interacción no permite que el discurso salga del control del profesor. Así, el alumno está logrando evidenciar su comprensión de los contenidos que son el objeto de su aprendizaje, pero tal comprensión se limita a la necesaria para mantener un dialogo que lidera el interlocutor, sin que se alcance a incorporar dentro de un discurso 
propio. Esto es, se ha logrado una comprensión inferencial, pero no se ha llegado aún a exhibir una competencia. Este objetivo del proceso de aprendizaje es precisamente el punto de la última de las aproximaciones que presentaremos.

\section{Tercera aproximación: lenguaje como una actividad de aprendizaje}

Se discutirá, finalmente, un tercer punto de vista que podría denominarse, siguiendo a Bronckart (2008), el socio-constructivismo discursivo. De acuerdo con esta perspectiva, la actividad del lenguaje, al ser una actividad de construcción de sentidos es esencialmente un proceso de producción de conocimientos. Así, la puesta en marcha de los mecanismos significantes y su realización en el discurso es, de hecho, un proceso cognitivo, es decir, de aprendizaje de contenidos. En palabras de Halliday (1993): “El lenguaje no es un dominio del conocimiento [...], el lenguaje es una condición para la cognición humana; es el proceso por medio del cual la experiencia se vuelve conocimiento". En consecuencia, y teniendo en cuenta que aquí 'aprender' es esencialmente asignar significados, una explicación del aprendizaje debe basarse en modelos de desarrollo de la competencia lingüística en el individuo. Esta posición, la más radical de las tres aquí anotadas, asigna al lenguaje el papel predominante en la actividad cognitiva humana y lo convierte, por tanto, en una actividad indispensable que incluso se confunde con el aprendizaje mismo.

El socio-constructivismo discursivo enfatiza sobre la forma en que el lenguaje en vez de reflejar pasivamente cierta estructura conceptual preexistente, tiene una participación activa en la aparición de tal estructura (Halliday y Martin, 1993; Wells, 1994). Para el asunto particular del aprendizaje escolar, Halliday y Martin nos recomiendan que debemos abandonar la noción de correspondencia ingenua entre el lenguaje y el conocimiento, y adoptar una perspectiva más constructivista. Las formas lingüísticas específicas de cierto dominio de conocimiento demuestran cómo éste no simplemente refleja o describe la experiencia humana, pues en vez de ello, la interpreta y la construye. Toda teoría científica, filosófica o experiencial es finalmente una construcción lingüística de la experiencia (p.ej., Lakoff y Johnson, 2001).

Esta mirada de construcción de conocimiento en el discurso tiene su origen en una visión de lenguaje como actividad al tiempo cognitiva y sociocultural que se remonta a los trabajos de Voloshinov (1977) y Bajtín (1984) sobre el fenómeno del discurso. Para la escuela soviética, el conocimiento humano tiene una condición esencialmente semiótica. Todo aquello que se conoce son signos de aspectos de la realidad que resultan ser sus referentes. Pero estos signos no surgen de la actividad individual solitaria, ya que son el resultado de discursos producidos en el marco de intercambios sociales. En esta medida, al tiempo que poseen un estatus psicológico, poseen una dimensión dialógica que los convierte en fenómenos sociales (Bronckart, 2008).

La perspectiva discursiva es entonces la perspectiva de un lenguaje en su contexto de uso sociocognitivo, en suma, una práctica socio-cultural. Así, todo enunciado, al ser producto de una situación de enunciación específica, la define (la situación) desde tres puntos de vista: 1) el de los contenidos que admite, 2) el de los recursos lingüísticos que permite y 3) el de las estrategias de organización discursivo cognitiva que reconoce como válidas (Bajtín, 1984).

Respecto de la definición de los contenidos debe decirse que, a través de los intercambios lingüísticos en el aula de clase, es necesario establecer, para cada situación, un ámbito de conocimiento desde el cual producir discursos posteriores, realizar todas las inferencias comunicativas relevantes y, en suma, saber de qué se está hablando. En otras palabras, una de las cosas que prácticamente siempre pasa en una clase es el establecimiento de límites sobre aquello de lo que se puede hablar o no hablar en dicha clase.

Un ejemplo que ilustra este primer propósito de aprendizaje, el de la definición de los contenidos que se admiten, puede observarse en la siguiente secuencia de clase. Se trata de una clase (Hefestos, 2 , 7) sobre el tema del origen de la vida y la evolución. Al inicio de la sesión, el profesor hace un sondeo de todas las posibles respuestas a la pregunta ¿Cómo 
se originó la vida en el planeta? que los estudiantes tenían de tarea. Después de algunas respuestas convencionales, una chica dice:

E4: Pues profe, yo consulté que... el origen de los organismos es un punto central de la "contención" (suponemos que la estudiante quiso decir "tensión") entre la creación y los hombres de la evolución.

P: O sea...

E4: O sea que la iglesia, cree que el origen de los animales es una creación divina y que los evolucionistas creen que fue obra de la naturaleza.

E: La evolución.

P: O sea que tú me estás diciendo que aquí hay dos puntos de vista en esto. Uno sería éste (señala en el tablero la palabra evolución) que sería el evolucionista y el otro sería el religioso, ¿cierto? Bien (escribe en el tablero la palabra religión) ¿Por cuál crees que nos vamos en la clase, ¿por éste? o ¿por este? (señala alternativamente las dos palabras).

E: Por éste.

E1: Por ese (justo cuando el profesor señala la palabra evolución).

P: ¿Por qué?

E1: Porque tiene unos criterios... dan una razón por la cual... y si eso está en una religión es como toda...

E2: Explican que Dios hizo todo eso.

E3: Existe y todo eso.

E4: Y no comprueban la...

E1: Pero no... no, porque no han, porque no han...

P: ¡Ah! Brian ${ }^{8}$ dice: pruebas, ¿cierto? O sea, aquí tenemos pruebas [... E Entonces me estaba diciendo Brian que hay unas pruebas que me pueden demostrar que esto es posible. Pero aquí, (señalando la palabra religión escrita en el tablero) difícilmente encontramos unas pruebas, ¿sí? Entonces...

Para el caso particular de la clase de ciencias naturales, una de las cosas que primero aprenden

8 Se refiere al niño arriba identificado como E4, quien dice "y no comprueban..." los estudiantes es a delimitar el campo conceptual de la disciplina, que corresponde, en esta clase, al ámbito científico. En la situación de aula, este campo de acción es igual al campo de la realidad de la que se puede hablar en clase. Queda claro en esta secuencia dialógica que en la clase de biología no se habla de religión ni de creacionismo, puesto que estos temas no son parcelas de conocimiento pertenecientes al conocimiento científico: “Por cuál crees que nos vamos en la clase?" y más tarde, "Entonces me estaba diciendo Brian que hay unas pruebas que me pueden demostrar que esto es posible. Pero aquí, (señalando la palabra religión escrita en el tablero) difícilmente encontramos unas pruebas, ¿si?"

Por otra parte, en lo que toca con la definición de los recursos lingüísticos que la situación permite, puede afirmarse que el aprendizaje incluye la apropiación de las formas lingüísticas adecuadas para expresar aquello de lo que se puede hablar en el aula. Así, parte de lo que debe ocurrir en una clase es el desarrollo de las estructuras formales del discurso por medio del cual una cierta porción de realidad es construida. Esto es necesario porque es en el proceso de adquisición de esas formas que se aprende una cierta forma de aproximación cognitiva a tal realidad.

Ilustraremos este segundo propósito de aprendizaje de definición de los recursos lingüísticos con un ejemplo de la clase sobre la organización molecular de la materia (Atenea, 2, 7). En esta parte de la clase, la profesora está presentando ejemplos de moléculas usando el código convencional para representarlas. Después de presentar la molécula del agua y la molécula del oxígeno, se sucede el siguiente intercambio:

P. Bueno, este es el tercero... de dióxido...moléculas de dióxido de carbono. Además de llamarse dióxido de carbono tiene otro nombre común, se llama gas carbónico, gas carbónico o dióxido de carbono. ¿De qué serán?, ¿Qué representarán los círculos rojos?

E. Oxígeno.

P. Átomos de [...] Dígame el nombre completo... Átomos de oxígeno. Y los negros? 
E. De carbono.

P. Átomos de carbono. De eso se puede descubrir la fórmula del dióxido de carbono, la fórmula química, como del agua es $\mathrm{H} 2 \mathrm{O}$, cómo será la fórmula química del dióxido de carbono. [...] Este es el modelo de las moléculas de dióxido de carbono. Los círculos rojos representan átomos de oxígeno, los negros representan átomos de carbono. Entonces cuál será la fórmula química de las moléculas de...,¿̨perdón?, del dióxido de carbono? Dígame, Ramírez

E. Dos moléculas de oxígeno y una de carbono.

P. Dos átomos de oxígeno y... dos átomos de oxígeno y uno de carbono, entonces la fórmula resulta... ¿Bocanegra?

E. $\mathrm{CHO} 2$ ?

P. H no porque aquí no hay átomos de hidrógeno, los átomos de hidrógeno los pintamos de azul, entonces acá $\mathrm{H}$ no, no hay átomos de hidrógeno.

E. Profe

P. Chaparro.

E. $\mathrm{CO} 2$

P. Se llama... CO2

Este fragmento de clase ilustra que al aprender un vocabulario y unas secuencias enunciativas concretas se está al tiempo aprendiendo una cierta forma particular de aproximarse a la realidad. En el ejemplo de arriba hay varios ejemplos de ello. $\mathrm{Al}$ inicio de la secuencia la profesora introduce el dióxido de carbono y lo "traduce" a su acepción en el lenguaje cotidiano: "Además de llamarse dióxido de carbono tiene otro nombre común, se llama gas carbónico". Posteriormente, la profesora pide explícitamente al estudiante que diga el nombre completo de uno de los componentes del modelo molecular que está presentando: "Átomos de... Dígame el nombre completo... Átomos de oxígeno”. Se está enfatizando aquí en la necesidad de manejar un registro lingüístico claro e inequívoco. Finalmente, la profesora trae a colación la necesidad de saber nombrar en un código especializado el compuesto químico que está representando a nivel molecular: "Entonces ¿cuál será la fórmula química de las moléculas [...], del dióxido de carbono?".
En el proceso de aprendizaje de estas formas lingüísticas, los niños de esta clase están aprendiendo que un mismo referente tiene tres posibles representaciones: gas carbónico, dióxido de carbono y CO2. Estas representaciones provienen de sistemas semióticos diferentes y cada uno de ellos posee reglas de "gramática" propias.

Finalmente, respecto a la definición de la organización cognitiva de la interacción específica, el discurso presente en el aula de clase tiene a su cargo la misión de inculcar una serie de principios organizativos implícitos en la construcción discursiva de una cierto tipo de saber. En la secuencia de clase a continuación (Hera, 2, 7), la profesora está explicando la diferencia entre compuestos iónicos y compuestos covalentes. Sus alumnos la acompañan con intervenciones muy cortas. Lo que interesa en este ejemplo es el discurso de la profesora:

P: ... hay diferencias entre los compuestos iónicos y los compuestos covalentes. Los compuestos covalentes tienen uniones estables pero no son $\tan$ fuertes...

E: Que los otros.

P: ... ellos alcanzan estabilidad electrónica y sus uniones son muy estables [...] En cambio, los enlaces iónicos son enlaces muy fuertes porque ¿qué pasa?... Acuérdense que allá teníamos un elemento que era electropositivo y otro electronegativo y las cargas diferentes, ¿qué les pasa??

E: Se unen.

E: Se atraen.

P: Se atraen. Como cuando tenemos dos imanes, ustedes lo vieron, ¿no? Dos imanes, cuando uno pone uno por el polo positivo y el otro, por el polo negativo, ¿qué siente uno? Cuando los va acercando... que se... se [ejerce] una fuerza de atracción, ¿cierto?

Esta secuencia discursiva muestra uno de los muchos momentos de esta clase en los que la profesora introduce en su discurso, alusiones a lo que podría considerarse evidencia empírica, como argumento

9 Se refiere a una clase previa en el laboratorio. 
para apoyar sus generalizaciones. Para ilustrar la atracción entre los polos de los compuestos iónicos, ella alude a una experiencia de laboratorio en la que los estudiantes "vieron" la atracción magnética. Mediante su discurso, la profesora está implicando que una manera de construir argumentos para apoyar afirmaciones sobre el mundo factual, desde un punto de vista científico, es hacer referencia a aquello que nuestra experiencia sensorial puede dar por hecho. Al ser el discurso de la profesora consecuente con los principios de validez para la epistemología convencional de las ciencias naturales está inculcando en sus estudiantes esa mirada "empírica" que sus discursos futuros habrán de tener.

Las situaciones de aula utilizadas para ilustrar la postura del socio-constructivismo discursivo ilustran por separado cada una de las tres condiciones que harían posible el aprendizaje por la vía de la acción lingüística. Debe decirse, sin embargo, que ninguna de ellas evidencia en su totalidad el ideal de la postura socio- discursiva. Para que una secuencia de clase lo hiciera, debería incluir un intercambio discursivo en el que un estudiante asumiera, de manera competente, las condiciones de delimitación temática, estructura formal y organización lógica del dominio de conocimiento que se intenta construir en la clase. Lamentablemente, no encontramos, en las 36 clases observadas, una porción de clase específica que ilustrara exactamente el logro ideal del planteamiento socio-discursivo. ¿Por qué?

Además de incluir la función representacional del lenguaje y ubicar el proceso de aprendizaje, en una situación de interacción comunicativa, esta última mirada a la relación lenguaje-conocimiento supone una exigencia adicional para que se logre su aplicación pedagógica: además de requerir la construcción conjunta de formas y significados, obliga también a la aparición de los mismos en el discurso del estudiante. Se trata éste de un ideal que apenas estaría iniciando su transposición a situaciones específicas en el aula de clase y que, a juzgar por la evidencia considerada, aún no tiene un peso importante en la acción pedagógica y didáctica.

\section{Conclusión}

A lo largo del presente escrito se ha intentado llegar a puntualizaciones sobre la relación entre lenguaje y conocimiento que vaya algo más allá del simple reconocimiento de su mutua interdependencia. Buscando estas precisiones hemos recorrido el camino de las teorías en las que se reconoce el papel fundamental del lenguaje para el aprendizaje y hemos ilustrado cada una de ellas mediante un ejercicio interpretativo de secuencias discursivas concretas en contextos de enseñanza y aprendizaje particulares.

A estas alturas, creemos que las claridades logradas han permitido apoyar la propuesta de Halliday (1993) en el sentido de que aprender cualquier cosa es como aprender una lengua. Pero entendamos todo aquello que supone aprender una lengua. Se trata de acercarnos a una realidad (empírica o construida) y aprender a significarla mediante prácticas enunciativas (un sistema semántico y sus correspondencias léxico-gramaticales); de desarrollar habilidades para la construcción conjunta de sentido con interlocutores que compartan los mismos sistemas de conocimientos, experiencias y valores (unas condiciones de uso del sistema en la comunicación) y de alcanzar, mediante el discurso, una praxis cognitiva socio-cultural reconocible por personas que realicen esa misma praxis (un género discursivo específico).

En este mismo sentido, pero desde la perspectiva de la enseñanza, Nussbaum y Tusón (1996) plantean que:

La clase de cualquier materia se asemeja a una clase de lengua extranjera. Quien enseña tiene que actuar como introductor o (re)presentador del discurso propio del aula y, más concretamente, del discurso específico de su área o ámbito de conocimiento. En cierto modo, las interacciones entre quien enseña y quien aprende se parecen a las que se producen entre nativos y no nativos. Quien enseña conoce, al menos parcialmente, las formas de hablar de quienes aprenden, pero quienes aprenden desconocen, a veces casi totalmente, 
las formas de hablar apropiadas en ese nuevo ámbito. Enseñar es en buena parte permitir el acceso a esas formas de hablar (léxico apropiado y específico, uso de conectores y de estructuras sintácticas determinadas, tipos de discurso, formas de participación, etc.). Para ello pueden y suelen utilizarse estrategias de facilitación para ayudar a la comprensión y a la elaboración de enunciados. Se trata de mecanismos de apoyo típicos de las conversaciones entre nativos y no nativos o de las llamadas interacciones exolingües (Nussbaum, 1991) en las que, aunque los hablantes utilicen el mismo código, recurren a variedades o registros que pueden estar muy alejados entre sí (Nussbaum y Tusón, 1996).

La analogía con la clase de lengua ha sido considerada seriamente en algunos contextos de las pedagogías específicas ${ }^{10}$. Mencionaremos como ejemplo la pedagogía de las ciencias, con los trabajos de Lemke (1990, 1997), Sutton (2003) y Mortimer

10 La analogía con el aprendizaje de una lengua no implica identificar la enseñanza con la simple imposición de un sistema arbitrario de rótulos verbales más o menos abstracto o más o menos especializado (por ejemplo, la jerga científica). Se trata, como en la actualidad se entiende el aprendizaje de una lengua, de asumir que todas las formas de conocimiento son actividades inmersas en entornos sociales y culturales definidos, y en esta medida están en constante construcción mediante procesos comunicativos y discursivos ( p.ej., De Longhi, 2000).
(2000), en los que se plantea la necesidad de incorporar la mirada discursivo-comunicativa en el desarrollo de modelos pedagógicos y didácticos para la enseñanza de las ciencias. Avanzando un poco más allá y asumiendo un enfoque culturalista para entender la situación de enseñanza y aprendizaje escolar (Cazden, 1991; Cole, 1997), de lo que se trataría, sería de inculcar en los estudiantes una práctica sociocultural, reflejada en un discurso (por ejemplo, el discurso de las ciencias y sus condiciones de producción) de manera tal que algo que era inicialmente ajeno a ellos llegue a ser parte de su repertorio interactivo-comunicativo cotidiano.

En este contexto resulta especialmente significativo, que haya sido en la aproximación socio-discursiva en la que tuvimos más dificultades para hallar una ilustración concreta dentro del corpus de las clases observadas. Ello estaría indicando que, en contraste con experiencias en contextos internacionales (véase especialmente Mortimer y Scott, 2003), la enseñanza escolar en el contexto colombiano se encuentra todavía poco permeado por los planteamientos del socio- constructivismo discursivo. Teniendo en cuenta su clara afinidad con la formulación de objetivos educativos en la forma de competencias, y su implementación pedagógica y didáctica es, sin duda, un propósito importante por lograr.h 


\section{Bibliografía}

Bajtín, M. M. (1984). "El problema de los géneros discursivos". En: M. M Bajtín, Estética de la Creación Verbal. México: Siglo XXI Editores S.A.

Best, J. (1999). Psicología Cognitiva. México: Paraninfo.

Boden, M.(1988). Computer models of the mind. Cambridge: Cambridge University Press.

Bonckart, J.P.(2005). Une introduction aux théories de laction. Geneve: Université de Geneve. Faculté de Psychologie et des Sciencies de L'Education.

Bronckart, J.P. (1997). “Teorías de la acción, lenguaje, lenguas naturales y discurso". En: Wertsch y otros (eds.) La mente sociocultural. Aproximaciones teóricas y aplicadas. Madrid: Fundación Infancia y Aprendizaje.

Bronckart, J.P. (2008). "Actividad lingüística y construcción de conocimientos". Lectura y Vida, vol 29, n. ${ }^{\circ}$, pp. 6-18.

Bruner, J. (1990). La Educación, Puerta de la Cultura. Madrid: Visor.

Candela, A. (1999). Ciencia en el aula. Los alumnos entre la argumentación y el consenso. México: Paidós.

Cazden, C. (1991). El discurso en el aula. El lenguaje de la enseñanza y el aprendizaje. Barcelona: Paidós.

Chomsky, N. y otros. (2002). El lenguaje y la mente humana. Barcelona: Ariel.

Cole, M. (1997). "La psicología sociocultural histórica algunos comentarios generales y una propuesta para una nueva metodología genético-cultural". En: Wertsch y otros (eds.) La mente sociocultural. Aproximaciones teóricas y aplicadas. Madrid: Fundación Infancia y Aprendizaje.

Coll, C. (1997). Aprendizaje escolar y construcción del conocimiento. Barcelona: Paidós.

Coll, C. y Onrubia, J. (2001). "Estrategias discursivas y recursos semióticos en la construcción de sistemas de significados compartidos entre profesor y alumnos". Investigación en la Escuela, n. ${ }^{\circ} 45$, pp.21-31.

Cook G., J. y Gumperz J. (1982). “Communicative Competence in Educational Perspective". En: Louise Cherry Wilkinson (ed) Communicating in the Classroom. New York: Academic Press.

Daniels, H. (2003). Vygotsky y la Pedagogía. Barcelona: Temas de Educación, Madrid: Paidós.

De Longhi, A. L. (2000). "El discurso del profesor y del alumno: un análisis didáctico en clase de ciencias". Enseñanza de las Ciencias, n. ${ }^{\circ} 18$, pp.201-216.
Ervin Tripp, S. (1982). "Structures of Control”. En: Louise Cherry Wilkinson (ed.). Communicating in the Classroom. New York: Academic Press.

Edwards, D. y Mercer, N. (1988). El conocimiento compartido: el desarrollo de la comprensión en el aula. Barcelona: Paidós.

Escandell, M. V. (1993). Introducción a la pragmática. Barcelona: Paidós.

Fodor, J. (1997). El Olmo y el Experto. El reino de la mente y su semántica. Barcelona: Paidós.

Fodor, J. (2000). La mente no funciona así. Alcance y límites de la psicología computacional. Madrid: Siglo XXI Editores.

Green, J. y Harker, J. (1982). “Gaining access to learning: Conversational, social and cognitive demands of group participation”. En: Louise Cherry Wilkinson (ed.). Communicating in the Classroom. New York: Academic Press.

Gutiérrez Rodilla, B. (1998). La ciencia empieza en la palabra. Análisis e historia del lenguaje científico. Barcelona: Península.

Halliday, M. K. y Martin, J. R. (1993). Writing science. Literacy and discursive power. London: Falmer Press.

Halliday, M.K. (1993). “Towards a language based theory of learning". Linguistics and Education, n. ${ }^{\circ}$ 5, pp. 93-116.

Lakoff, G. y Johnson,M. (2001). Metáforas de la vida cotidiana. Madrid: Cátedra.

Leech, G. (1983). Principles of pragmatics. London: Longman Linguistics Library.

Lemke, J. (1988). "Genres, semantics and classroom education". Linguistics and Education, n. ${ }^{\circ} 1$, pp. 81-99.

Lemke, J. (1997). Aprender a hablar ciencia. Lenguaje, aprendizaje y valores. Barcelona: Paidós.

Levinson, S. (1985). Pragmatics. Cambridge: Cambridge Texbooks in Linguistics.

Martin, R.J. (1993). Genre and literacy. Modelling contexts in educational linguistics. Sidney: University of Sidney Press.

Matthews, P.H. (1983). Gramática Generativa y Competencia Lingüística. Madrid: Espasa.

Mercer, N. (2001). Palabras y mentes. Cómo usamos el lenguaje para pensar juntos. Barcelona: Paidós.

Mortimer, E. y Scott, P. (2003). Meaning making in secondary science classes. Berkshire: McGraw Hill. 
Mortimer, E. (2005). Lenguaje y formación de conceptos en la enseñanza de las ciencias. Madrid: A. Machado Libros, S.A.

Mortimer, E. y Wertsch, J. (2003). The architecture and dynamics of intersubjectivity in science classrooms, mind, culture and activity, vol. 10, n. ${ }^{\circ 3}$, pp. 230-244.

Nussbaum, L. y Tusón, A. (1996). El aula como espacio cultural y discursivo. Signos, Teoría y Práctica de la Comunicación, n. ${ }^{\circ}$ 17, Enero-Marzo, pp.14-21.

Paivio, A. (1986). Mental representations. Oxford: Oxford University Press.

Rickenmann, R. (2007). Metodologías clínicas de investigación en didácticas y formación del profesorado: un estudio de los dispositivos de formación en alternancia. Conferencia Seminario Doctoral.

Rogoff, B. (1997). Los tres planos de la actividad sociocultural: apropiación participativa, participación guiada y aprendizaje. En: Wertsch y otros (eds.) La mente sociocultural. Aproximaciones teóricas y aplicadas. Madrid: Fundación Infancia y Aprendizaje.

Rumelhart, D. y Ortony, A. (1977). “The representation of knowledge in memory". En: R. Anderson y otros (eds.). Schooling and the acquisition of knowledge. Chichester: Wiley.

Schank, R. y Abelson, R. (1977). Guiones, planes, metas y entendimiento. Barcelona: Paidós.

Sperber, D. (1994). "Understanding verbal understanding". En: Jean Khalfa (ed.). What is Intelligence?, pp. 179-198. Cambridge University Press.
Sperber, D. \& Deirdre, W. (2006). "Pragmatics”. En: F. Jackson y M. Smith (eds.). Oxford Handbook of Philosophy of Language.

Sperber, D. \& Wilson,D. (1995). Relevance: Communication and Cognition. Oxford: Blackwell.

Stubbs, M. (1983). Language, schools and classrooms. London: Metheun.

Sutton, C. (2003). "Los profesores de ciencias como profesores de lenguaje”. Enseñanza de las Ciencias, vol, 21, n. ${ }^{\circ}$, pp. 21-25.

Tuson, A. (1997). Análisis de la conversación. Barcelona: Editorial Ariel.

Voloshinov, V.N. (1977). Marxism et philosophie du langage. Paris: Minuit (Edición original de 1929).

Vygostky, L. (1979). Pensamiento y Lenguaje. Buenos Aires: Pléyade.

Vygotsky, L. (1979). El desarrollo de los procesos psicológicos superiores. Madrid: Crítica.

Wells, G. (1994). “The Complementary Contributions of Halliday and Vygotsky to a Language based theory of Learning". Linguistics and Education, vol.6, pp.4190.

Wilkinson, L. C. (1982). "A Sociolinguistic approach to communicating in the classroom". En: L.C. Wilkinson (ed.). Communicating in the classroom, pp 3-10. London: Academic Press.

Wood, D., Bruner, J. y Ross, G. (1976). “The role of tutoring in problem solving". Journal of Child Psychology and Psychiatry, vol. 17, pp.89-100. 
